\section{Descentralização das ações de vigilância epidemiológica em Pernambuco, Brasil}

\author{
Decentralization of epidemiological surveillance \\ in Pernambuco State, Brazil
}

\author{
Descentralización de la vigilancia epidemiológica \\ en Pernambuco, Brasil
}

\author{
${ }^{1}$ Instituto de Saúde Coletiva, \\ Universidade Federal da \\ Bahia, Salvador, Brasil. \\ 2 Secretaria Executiva \\ de Vigilância em Saúde, \\ Secretaria de Saúde do \\ Estado de Pernambuco, \\ Recife, Brasil. \\ 3 Grupo de Estudos de \\ Gestão e Avaliação em \\ Saúde, Instituto de Medicina \\ Integral Professor Fernando \\ Figueira, Recife, Brasil. \\ Correspondência \\ A. C. Albuquerque \\ Rua Taquaritinga 149, apto. \\ 60, Recife, $P E$ \\ 52070-649, Brasil. \\ ana_albuquerque1@hotmail.com
}

Ana Coelho de Albuquerque 1,2 Eduardo Luiz Andrade Mota 1 Eronildo Felisberto ${ }^{3}$

\section{Resumo}

The study aimed to analyze the relationship between decentralization of the Brazilian Unified National Health System (SUS) and the development of epidemiological surveillance activities in municipalities (counties) in Pernambuco State, Brazil. This was an exploratory descriptive qualitative and quantitative study, including a document search, completion of semi-structured interviews by key informants, and an ecological spatial and time trend study of selected health indicators, covering a 10-year period (20012010). The study showed that municipalities adhered to the decentralization process, which was making progress in Pernambuco, but with inequalities and weaknesses in its development. There was also a fluctuation in the time series for the selected indicators. Thus, even though the decentralization of epidemiological surveillance is still incipient in some municipalities, their protagonist role in implementing the activities promotes empowerment at the local level by producing key information for decision-making.

Epidemiological Surveillance; Decentralization; Municipal Management
O objetivo do estudo foi analisar a relação entre o processo de descentralização da gestão do Sistema Único de Saúde (SUS) e o desenvolvimento das ações de vigilância epidemiológica em municípios de Pernambuco, Brasil. A estratégia de trabalho consistiu num estudo descritivo e exploratório qualitativo/quantitativo, com a realização de uma pesquisa de caráter documental e entrevistas semiestruturadas com informanteschave, como também um estudo ecológico espacial e de série temporal, no qual foi traçada uma série histórica de dez anos (2001-2010), utilizando-se indicadores selecionados. O estudo evidenciou que o processo de descentralização em Pernambuco obteve adesão e evoluiu, porém com a existência de desigualdades e fragilidades no seu desenvolvimento, também apontadas pela oscilação nos resultados da série histórica dos indicadores selecionados. Assim, mesmo que o processo de descentralização das ações de vigilância epidemiológica ainda seja incipiente em alguns municípios, sabe-se que o papel de executor das ações promove o empoderamento do nível municipal, quando o mesmo passa a produzir as informações necessárias à tomada de decisão.

Vigilância Epidemiológica; Descentralização; Administração Municipal 


\section{Introdução}

O aperfeiçoamento do modelo organizacional do Sistema Único de Saúde (SUS) tem levado à racionalização de responsabilidades entre instâncias gestoras, que decorre do reconhecimento do município como o espaço privilegiado para tratar das questões setoriais. Assim, em cumprimento dos princípios constitucionais do SUS implantou-se o processo de descentralização, a partir da década de 1990, como a estratégia central de rearranjo organizacional, com a transferência para os municípios da responsabilidade e dos recursos para o exercício e gestão dos serviços de saúde prestados em seu território 1 . Inicialmente, esse processo concentrou-se na reorganização da gestão da assistência à saúde. A descentralização das ações de vigilância epidemiológica aconteceu posteriormente, com a edição da Norma Operacional Básica 01/96 (NOB 01/96) e, em dezembro de 1999, a partir da publicação das Portarias Ministeriais no 1.399 e 950, que regulamentaram o papel de cada gestor na organização das ações de epidemiologia, prevenção e controle de doenças 2,3 .

Sabe-se que, embora o processo de descentralização da gestão da saúde atinja hoje quase a totalidade dos municípios brasileiros, o mesmo se desenvolveu de forma assimétrica e diversificada ao longo do tempo e entre as diversas regiões do país, em que a qualidade das ações e a efetividade dos resultados são desafios ainda presentes. O Sistema Nacional de Vigilância Epidemiológica (SNVE), como elemento estruturante do SUS, está inserido nessa realidade, na qual é notória a variação na implantação e na descentralização de suas ações, razão porque o estudo desse processo e do seu impacto necessita de novas pesquisas. Devido às diferenças políticas regionais, alguns municípios avançam mais do que outros na implantação das ações descentralizadas, em um contexto de recursos escassos ou subutilizados em muitos deles. Em consequência, ações incipientes e de baixo impacto na situação de saúde da população são operacionalizadas, pois se considera que a maioria dos municípios ainda não está estruturada para assumir efetivamente todos os encargos da descentralização da vigilância 4 .

O uso efetivo e sistemático das informações da vigilância epidemiológica é uma ferramenta imprescindível, tanto na gestão do sistema de saúde para a definição de prioridades e alocação de recursos, quanto para nortear as estratégias de intervenção dos serviços 5 . Desde o início do processo de construção e implantação do SUS foi debatida uma proposta de vigilância coerente com a necessidade de reorganizar e reorientar os serviços de saúde no país 6 . A integração entre as ações típicas de vigilância na execução dos programas de prevenção e controle de doenças suscitou intensos debates em determinados períodos da história sanitária recente do país, ao mesmo tempo em que produziu e legitimou práticas concretas nos sistemas locais de saúde 7 .

Nesse contexto, apresentam-se os resultados de um estudo que objetivou analisar a relação entre o processo de descentralização da gestão do SUS e a operacionalização das ações de vigilância epidemiológica em municípios de Pernambuco, no período de 2001 a 2010. Assim, a temática abordada por este artigo tem o intuito de contribuir com elementos para o aperfeiçoamento da descentralização da vigilância epidemiológica nos níveis estadual e municipal, e oferecer subsídios para o planejamento e para a programação de ações que visem a resultados efetivos, bem como para a definição de prioridades na alocação de recursos na área de vigilância epidemiológica dos sistemas municipais de saúde.

\section{Métodos}

Realizou-se um estudo descritivo e exploratório que utilizou duas abordagens para o alcance de seu objetivo. Considerando a complexidade e a extensão do objeto estudado, a estratégia metodológica foi organizada em dois momentos: no primeiro, com enfoque qualitativo, foi realizada uma pesquisa documental e entrevistas semiestruturada com informantes-chave; no segundo momento, em um estudo de cunho quantitativo, de desenho ecológico espacial e de série temporal, foi traçada uma série história de dez anos (2001-2010) utilizando-se indicadores extraídos do Painel de Monitoramento do Desempenho da Gestão da Secretaria Executiva de Vigilância em Saúde da Secretaria de Saúde do Estado de Pernambuco (SES-PE) 8 .

As unidades de análise do estudo foram os dez municípios-sede das Gerências Regionais de Saúde (Geres) de Pernambuco, uma amostra intencional na qual se considerou que estes municípios revelavam as características específicas de cada mesorregião do estado. A ausência no estudo dos municípios-sede da XI e XII Geres justifica-se pelo fato de as mesmas só terem sido criadas nos anos de 2006 e 2012, respectivamente.

$\mathrm{Na}$ pesquisa documental e nas entrevistas com os informantes-chave foram contempladas as seguintes categorias de análise: período em que foi realizado o processo de descentralização das ações de vigilância epidemiológica; extensão da descentralização; evolução temporal e espacial da descentralização com relação à estrutu- 
ração da vigilância epidemiológica (recursos humanos, físicos e materiais); fatores facilitadores e dificultadores da descentralização da vigilância epidemiológica; fatores positivos e negativos do processo de descentralização para o nível municipal; repasse de recursos financeiros para a vigilância epidemiológica; e integração das ações de vigilância epidemiológica com a Estratégia Saúde da Família (ESF).

Com relação às entrevistas, os gestores foram selecionados intencionalmente de acordo com os objetivos do estudo. Nos dez municípios-sede das Geres foram realizadas entrevistas com todos os gestores de vigilância epidemiológica ou de vigilância em saúde e em três municípios também com o Secretário de Saúde. Utilizou-se como critério de seleção a participação no processo de descentralização das ações de epidemiologia e controle de doenças nos municípios correspondentes. Foram também entrevistados os gestores estaduais de vigilância em saúde ou vigilância epidemiológica em $50 \%$ das Geres, utilizando-se o mesmo critério de seleção. Na pesquisa de caráter documental foram usadas, como fontes de dados, as resoluções da Comissão Intergestores Bipartite de Pernambuco (CIB-PE) e os protocolos estaduais para certificação da descentralização das ações de vigilância epidemiológica.

A análise dos dados oriundos da pesquisa documental e das entrevistas foi baseada na técnica de análise de conteúdo que se apoia na codificação da informação em categorias, de forma a dar sentido ao material estudado. Essa análise de conteúdo foi composta por três fases: a préanálise, que abrangeu a escolha do material, a formulação de hipóteses e objetivos e a elaboração de indicadores para a interpretação dos resultados; a análise do material, que consistiu na codificação, categorização e quantificação da informação; e o tratamento dos resultados que envolveu procedimentos diversos 9 .

Após a leitura exaustiva do material utilizado como fonte documental, foram identificados fatos ou informações que se relacionaram com as ações de implementação da descentralização da vigilância epidemiológica, de acordo com as categorias analíticas já descritas. Nas entrevistas com os informantes-chave foi realizada análise do tipo temática que, segundo Minayo 10 (p. 209), consiste "em descobrir os núcleos de sentido que compõem uma comunicação cuja presença ou frequência signifiquem alguma coisa para o objetivo analítico visado". Os dados considerados relevantes, referentes às categorias analíticas, foram inseridos de forma ordenada e cronológica em uma matriz de análise.

Para o estudo exploratório de desenho ecológico foi realizada uma seleção de indicadores 8 que representassem ações inerentes ao nível municipal e, portanto, fossem indicativos do grau de responsabilização do município a partir da implantação das ações descentralizadas, como também tivessem dados disponíveis para seu cálculo na série histórica do estudo. Assim, foram selecionados os seguintes indicadores: proporção de casos novos de tuberculose pulmonar do tipo bacilífero encerrados pelo critério de cura, proporção de óbitos com causa básica definida e taxa de incidência de sífilis congênita. Esse último indicador substituiu o indicador número de casos de sífilis congênita do Painel de Monitoramento citado.

Para o cálculo desses indicadores foram utilizados dados secundários, disponibilizados pela SES-PE. Os dados referentes à população dos municípios do estudo foram obtidos das bases digitais disponibilizadas na página de Internet do Instituto Brasileiro de Geografia e Estatística (IBGE; http://www.ibge.gov.br). Para a tabulação foi elaborado um banco de dados único em planilha Excel (Microsoft Corp., Estados Unidos), com os dados brutos e os indicadores de interesse. Para descrever a evolução temporal dos indicadores selecionados foram utilizadas tabelas, nas quais se relacionou o tempo (em anos) com os municípios de acordo com o resultado de cada indicador. Além disso, foi verificada a variação percentual entre o ano inicial e final do período do estudo (2001-2010) de cada município e do estado.

Seguindo as recomendações da Resolução no 196/96 da Comissão Nacional de Ética em Pesquisa (CONEP), o estudo foi aprovado pelo Comitê de Ética em Pesquisa do Instituto de Saúde Coletiva da Universidade Federal da Bahia, por meio do registro no 031-12/CEP-ISC, estando de acordo com os princípios éticos para a pesquisa que envolve dados primários baseados em pesquisa documental e entrevistas, como também dados secundários.

\section{Resultados}

\section{Evolução temporal e espacial da descentralização da vigilância epidemiológica}

Observou-se que o ano 2000 marcou o início do processo de certificação dos sistemas municipais de vigilância epidemiológica em Pernambuco, encontrando-se todos eles habilitados com base na NOB 01/96. Nesse ano, do total de 184 municípios e o território de Fernando de Noronha, 92\% ( $\mathrm{n}=169)$ estavam habilitados na gestão plena da atenção básica e $8,1 \%(n=15)$ estavam 
habilitados na condição de gestão plena do sistema municipal de saúde 11. É possível perceber a adesão progressiva das Secretarias Municipais de Saúde (SMS) ao processo de certificação, desde a publicação dos atos normativos em dezembro de 1999 até dezembro de 2011. Em 2000, apenas $15(8,1 \%)$ municípios pernambucanos foram certificados, já em 2011 este número atingiu 181 $(97,8 \%)$ (Figura 1).

Demonstrou-se a existência de três fases distintas na evolução temporal do processo de descentralização da gestão da vigilância epidemiológica em Pernambuco (Figura 1). O primeiro momento está compreendido entre os anos de 2000 e 2003, quando se observou um crescimento expressivo do número de municípios certificados (de $8,1 \%$ para $66,3 \%$, um incremento de $818,5 \%$ ); o segundo, entre os anos de 2003 e 2006 , no qual se notou uma fase de pouca atividade desse processo, quando apenas $3,8 \%$ dos municípios foram certificados para exercer as ações de epidemiologia e controle de doenças; e por fim, após o ano de 2006, tem-se o terceiro e último momento, no qual as certificações voltaram a acontecer de forma acentuada.

Com relação à evolução espacial, nota-se que em Pernambuco não ocorreu um padrão específico de disseminação da implantação da descentralização da vigilância epidemiológica, porém é possível perceber algumas características importantes deste processo. No primeiro ano das certificações, apenas três municípios-sede das Geres foram contemplados, acontecendo a descentralização em todos os municípios-sede nos anos subsequentes, entre 2001 e 2003. Em todas as Geres e mesorregiões, ocorreram certificações de municípios logo no primeiro triênio do período estudado (Figura 2).

Destaca-se o caso da I Regional, onde estão localizados os municípios da Região Metropolitana de Recife, na qual em todos eles houve a implantação da descentralização da vigilância epidemiológica, e o caso da VII Regional (Sertão), onde apenas um município não foi certificado no período de 2001 a 2003. Nota-se também nos mapas que apenas três municípios pernambucanos ainda não estavam habilitados para exercer as ações de vigilância epidemiológica ao final do período estudado (2011), sendo dois deles pertencentes à V Regional (Agreste) e um deles àVI Regional (Sertão) (Figura 2).

\section{A percepção dos gestores com relação ao processo de descentralização da vigilância epidemiológica}

Em 70\% dos municípios estudados os gestores apontaram a Portaria Ministerial no 1.399 2, publicada em 16 de dezembro de 1999, como o marco do processo de descentralização das ações de vigilância e controle de doenças. Essa Portaria, que regulamentou a NOB 01/96 no que diz

Número acumulado de municípios certificados para ações de epidemiologia e controle de doenças, por ano de certificação. Pernambuco, Brasil, 1999-2011.

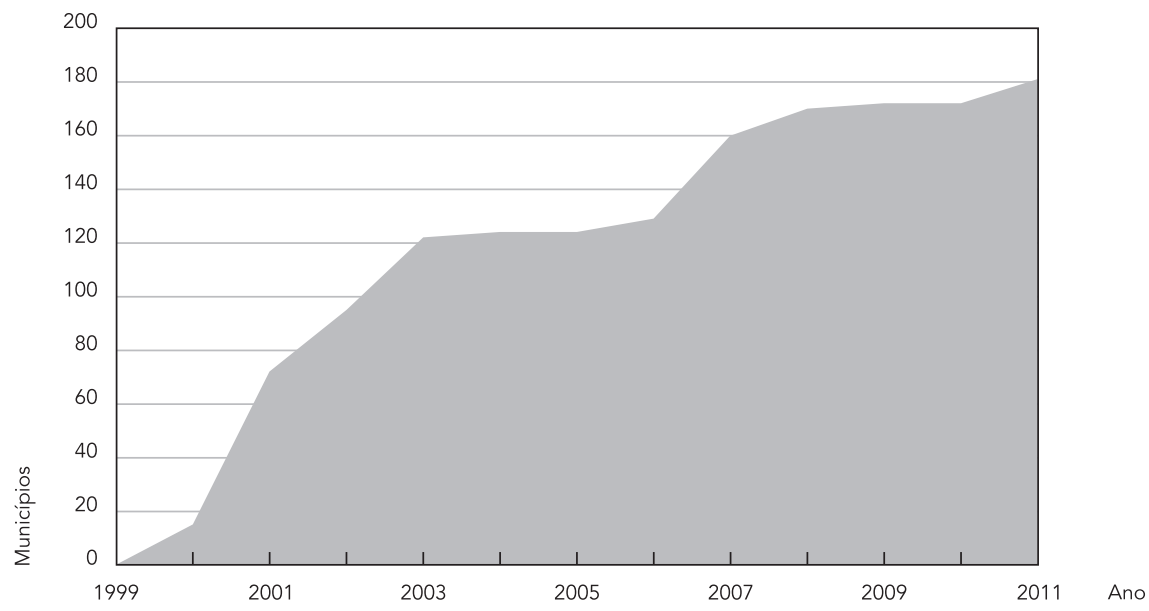




\section{Figura 2}

Municípios certificados para as ações de epidemiologia e controle de doenças. Pernambuco, Brasil, 2000-2011.

2a) 2000

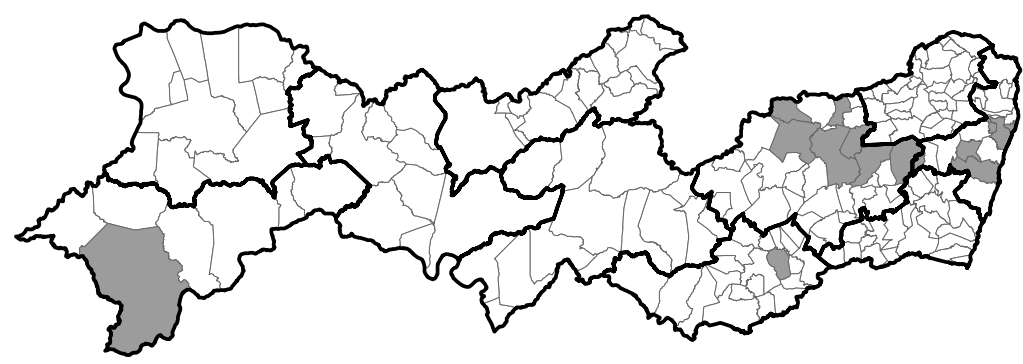

2b) $2000-2003$

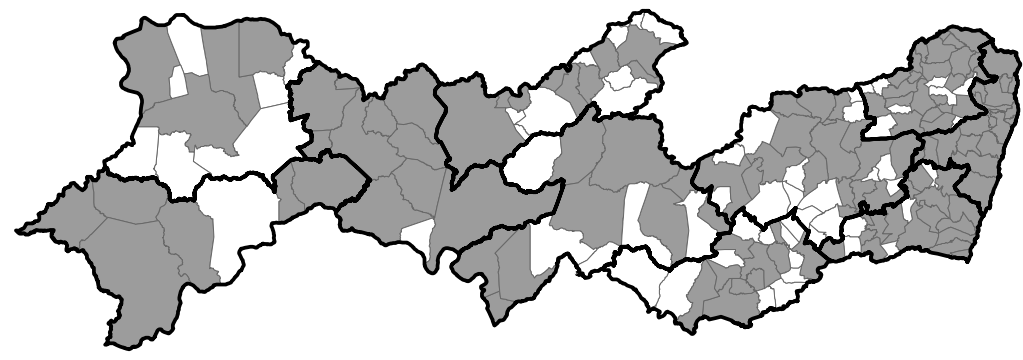

2c) $2000-2006$

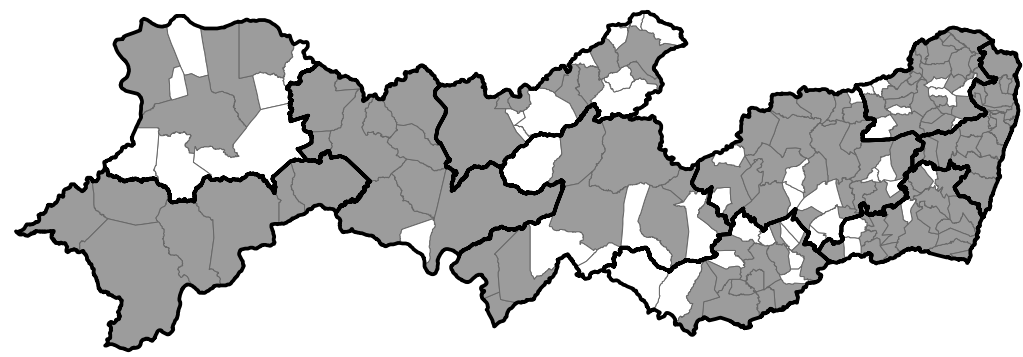

2d) $2000-2011$

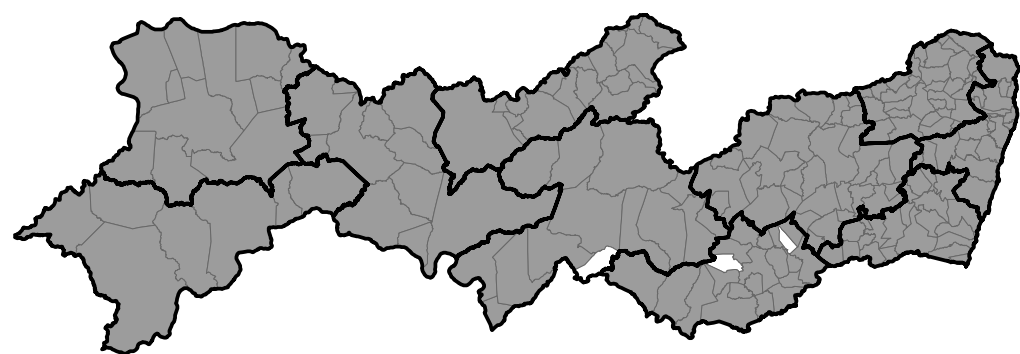

Municípios com certificação

Municípios sem certificação

$0 \frac{n_{s}^{N}}{k^{k}}$

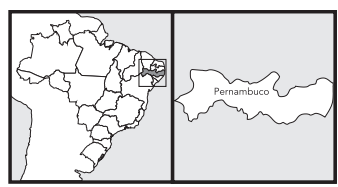


respeito à sistemática do financiamento, segundo aqueles gestores, favoreceu o interesse dos municípios pela certificação, uma vez que o recurso do Teto Financeiro de Epidemiologia e Controle de Doenças (TFECD) passou a ser transferido diretamente do Fundo Nacional de Saúde para o Fundo Municipal de Saúde (FMS), a partir da sua implantação. Assim sendo, nota-se pelos relatos dos gestores, que o incentivo financeiro foi o principal impulsionador do processo de descentralização da vigilância epidemiológica em Pernambuco. Em alguns municípios estudados também foi citado como fator que contribuiu para o processo de descentralização a vontade política do gestor municipal.

Com relação à evolução da descentralização da vigilância epidemiológica nos municípios, em todas as entrevistas observou-se o relato de desigualdades no processo. Em 30\% dos municípios estudados os gestores apontaram que, mesmo após o processo de descentralização, a vigilância epidemiológica ainda executa de forma insatisfatória algumas ações como a investigação de óbitos e análise e disseminação das informações. Já no que diz respeito ao quadro de recursos humanos e à estrutura física, 90\% dos gestores destacaram que houve uma evolução significativa. Um gestor considerou que, apesar desses avanços, alguns aspectos ainda inibem o crescimento da vigilância epidemiológica. A insuficiência e o despreparo da equipe técnica, bem como o organograma "engessado" das SMS não acompanharam o processo de implantação da descentralização da vigilância epidemiológica, o que promoveu o aumento das atribuições do nível municipal. O mesmo gestor apontou que, no seu município, a estruturação de uma equipe técnica capacitada contribuiu para que a vigilância epidemiológica evoluísse, alcançando seu espaço junto à Gestão Municipal.

Cabe ressaltar, no entanto, que o processo de implantação da descentralização das ações de vigilância epidemiológica nos municípios aconteceu à proporção que os gestores municipais sinalizavam o interesse em certificar-se, assumindo que contavam com a estrutura mínima definida pelo instrumento de certificação. Porém, na fala da maioria dos gestores foi possível observar que a realidade era outra, posto que faltavam profissionais qualificados, estrutura física, insumos (computadores, impressoras) e veículos na vigilância epidemiológica à época. Em $80 \%$ deles, até o momento das entrevistas, a falta de veículos configurava um aspecto dificultador da execução das ações. Acrescenta-se que um dos fatores apontados pelos gestores como dificultador da implantação da descentralização da vigilância epidemiológica foi o desconhecimento, por par- te dos gestores e técnicos, acerca da importância e do papel da vigilância epidemiológica como parte integrante do sistema de saúde municipal. Disso resultou, como foi apurado nos relatos, que no início do processo de descentralização ocorreu grande perda na qualidade da informação, pois os municípios não faziam a crítica adequada com relação às inconsistências e incompletitudes existentes nas notificações de casos. Os municípios assumiram a tarefa de notificar, digitar, registrar e investigar os casos, ações que antes eram executadas no nível regional ou central da SES, mas devido à fragilidade e ao despreparo técnico de suas equipes, os dados muitas vezes não tinham robustez.

Outro ponto citado nas entrevistas foi a forma verticalizada com que o processo de certificação dos municípios foi instituído, do nível federal para o estadual e, principalmente, para o municipal o que, segundo os relatos, contribuiu de forma direta com o fato citado. Esse relato foi ratificado em várias entrevistas e enfatizado por um gestor que ainda destacou que as ações eram pactuadas pelos municípios na Programação Pactuada Integrada de Epidemiologia e Controle de Doenças (PPI-ECD), vindas do Ministério da Saúde como "um pacote pronto a ser cumprido", sem que fossem observadas as particularidades de cada um.

No relato da totalidade dos gestores entrevistados, a descentralização das ações da vigilância epidemiológica para o nível municipal trouxe como aspecto positivo a aproximação do nível executor das ações à população e seus problemas de saúde. Para o Gestor F, essa aproximação resulta numa maior responsabilização por parte da gestão municipal, em relação ao periodo anterior. Além disso, para o Gestor Q o papel de executor das ações promove o empoderamento do nível municipal, pois o mesmo passa a produzir as informações necessárias à tomada de decisão.

Com relação ao financiamento, os gestores apontaram que a criação do FMS facilitou o processo de implantação da descentralização, quando o gestor do recurso passou a ser o próprio Secretário de Saúde do município. No que diz respeito à criação do TFECD (atualmente denominado de Piso Financeiro de Vigilância em Saúde - PFVS), destinado ao custeio das ações da vigilância epidemiológica, os gestores também destacaram a importância do mesmo no processo de implantação da descentralização. Mesmo naqueles municípios onde o movimento descentralizador da vigilância epidemiológica ocorreu antes mesmo da publicação da Portaria no 1.399/99, os gestores entrevistados relataram que o repasse dos recursos por meio do TFECD contribuiu ainda mais com o seu avanço. Em 90\% dos municípios do estudo, a administração 
do FMS, bem como do PFVS era feita pela SMS, sendo o Secretário de Saúde o gestor. No entanto, foi possível observar que essa realidade não era a mesma para todos os municípios. O Gestor $C$ relatou que, apesar de ser oficialmente o gestor do Fundo (e, consequentemente, do PFVS), na prática esse recurso era administrado pelo setor financeiro ligado diretamente ao gabinete da Prefeitura.

Por fim, $60 \%$ dos gestores entrevistados relataram a existência de integração entre a vigilância epidemiológica e a ESF nos seus municípios. Para o Gestor R essa integração foi condição imprescindível à descentralização das ações da vigilância epidemiológica, uma vez que a ESF cumpre com o papel de executor das ações básicas de saúde no nível local, sendo a notificação e a investigação exemplos dessas ações. Esse Gestor destacou ainda, como uma experiência exitosa, o fato da ESF ter sido implantada no seu município com base no perfil epidemiológico local, mostrando assim a importância da vigilância epidemiológica para a gestão municipal. Em contraste, o Gestor $\mathrm{G}$ apontou a dificuldade de se obter essa integração, dando como exemplo a centralização dos programas de controle da hanseníase e da tuberculose executados pela própria vigilância epidemiológica no nível central da SMS e na unidade de referência. Nesse município, as unidades da ESF realizavam apenas algumas ações de educação em saúde, como palestras, ficando a cargo da vigilância epidemiológica toda a parte de acompanhamento dos pacientes e dispensação de medicamentos. A fala do Gestor P também corrobora a importância da integração entre a vigilância epidemiológica e a ESF, mas relata que a mesma não acontece de forma regular no seu município, sendo observada apenas em momentos de crise e epidemias, como por exemplo, no enfrentamento da epidemia de dengue.

\section{A evolução de indicadores da vigilância epidemiológica no processo de descentralização}

No que se refere ao indicador proporção de casos novos de tuberculose pulmonar bacilíferos encerrados pelo critério de cura, observou-se o crescimento de $60 \%$ nos municípios estudados, resultado este que contrasta com o que se verificou no Estado de Pernambuco, cuja variação percentual demonstrou uma diminuição do valor deste indicador $(-6,3 \%)$ no mesmo período. Nota-se, no entanto, que esse crescimento não aconteceu de modo contínuo em todos os anos da série. Pode-se destacar o Município de Garanhuns, com aumento de $71,4 \%$, sendo a maior variação percentual positiva dentre os municípios da pesquisa, e Caruaru, com apenas 2,3\%, a menor variação positiva, e ainda os municípios de Ouricuri e Salgueiro, que obtiveram 94,4\% e $92,3 \%$, respectivamente, de casos encerrados por cura em 2010 (Tabela 1).

Para o indicador proporção de óbitos com causa básica definida, notou-se que em $100 \%$ dos municípios pesquisados houve um aumento dos

Proporção de casos novos de tuberculose pulmonar bacilíferos encerrados pelo critério de cura e variação percentual, segundo município de residência. Pernambuco, Brasil, 2001-2010.

\begin{tabular}{|c|c|c|c|c|c|c|c|c|c|c|c|}
\hline \multirow[t]{2}{*}{ Municípios } & \multicolumn{10}{|c|}{ Ano } & \multirow{2}{*}{$\begin{array}{l}\text { Variação (\%) } \\
2001-2010\end{array}$} \\
\hline & 2001 & 2002 & 2003 & 2004 & 2005 & 2006 & 2007 & 2008 & 2009 & 2010 & \\
\hline Recife & 72,3 & 65,9 & 67,2 & 68,5 & 71,4 & 69,6 & 71,3 & 71,9 & 65,6 & 63,4 & $-12,3$ \\
\hline Limoeiro & 90,0 & 91,7 & 85,7 & 77,3 & 66,7 & 87,5 & 78,6 & 76,5 & 86,2 & 78,6 & $-12,7$ \\
\hline Palmares & 76,9 & 73,3 & 54,5 & 66,7 & 72,0 & 85,0 & 78,1 & 81,0 & 80,8 & 85,2 & 10,7 \\
\hline Caruaru & 64,3 & 75,0 & 59,5 & 63,9 & 68,8 & 77,8 & 71,4 & 55,9 & 59,3 & 65,8 & 2,3 \\
\hline Garanhuns & 50,0 & 75,0 & 75,0 & 75,0 & 76,9 & 92,0 & 50,0 & 85,7 & 66,7 & 85,7 & 71,4 \\
\hline Arcoverde & 55,6 & 50,0 & 76,9 & 90,9 & 69,2 & 66,7 & 50,0 & 83,3 & 70,0 & 62,5 & 12,5 \\
\hline Salgueiro & 81,8 & 77,8 & 66,7 & 90,0 & 76,9 & 63,2 & 78,6 & 70,0 & 100,0 & 92,3 & 12,8 \\
\hline Petrolina & 81,3 & 64,7 & 72,7 & 89,7 & 82,1 & 83,3 & 73,3 & 63,0 & 53,3 & 59,3 & $-27,1$ \\
\hline Ouricuri & 66,7 & 50,0 & 60,0 & 91,7 & 88,9 & 90,9 & 91,7 & 91,7 & 100,0 & 94,4 & 41,7 \\
\hline Afogados da Ingazeira & 80,0 & 33,3 & 100,0 & 100,0 & 0,0 & 100,0 & 100,0 & 83,3 & 75,0 & 66,7 & $-16,7$ \\
\hline Total no estado & 72,5 & 71,2 & 69,8 & 73,3 & 72,4 & 73,3 & 74,2 & 72,1 & 70,6 & 67,9 & $-6,3$ \\
\hline
\end{tabular}

Fonte: Sistema de Informação de Agravos de Notificação (SINAN)/Secretaria Executiva de Vigilância em Saúde da Secretaria de Saúde do Estado de Pernambuco. 
seus valores, com ênfase para Ouricuri e Afogados da Ingazeira, com $92 \%$ e $75,8 \%$ de aumento, respectivamente. Em $60 \%$ dos municípios a variação observada foi maior do que aquela obtida no estado (20,1\%). É possível perceber ainda uma evolução progressiva dos valores desse indicador nos anos da série histórica estudada, com algumas exceções. Nota-se que, no ano de 2001, sete municípios tinham menos de $80 \%$ dos seus óbitos com a causa básica definida, e que destes, seis atingiram $90 \%$ ou mais no final do período estudado (Tabela 2).

Com relação à taxa de incidência de sífilis congênita, vê-se que em $40 \%$ dos municípios houve diminuição, destacando-se Limoeiro e Arcoverde, com variação percentual de $-100 \%$ de 2001 para 2010. Quatro municípios acompanharam a tendência no estado, que também apresentou diminuição da taxa de incidência de sífilis congênita (-3,3\%). Em $20 \%$ da amostra, o valor desse indicador foi zero em quase todos os anos da série histórica. Tanto nos municípios que apresentaram diminuição quanto naqueles que apresentaram aumento, observou-se uma oscilação nos dados, com momentos de crescimento e de diminuição em quase todos eles, destacando-se Limoeiro e Petrolina, nos quais a maior taxa de incidência de sífilis congênita foi de 8,8/1.000 nascidos vivos em 2002 e 7,5/1.000 nascidos vivos em 2001, respectivamente, e a menor taxa foi de 0,0/1.000 nascidos vivos em 2010 e $1,3 / 1.000$ nascidos vivos em $2008 / 2009$, respectivamente (Tabela 3 ).

\section{Discussão}

A pesquisa documental e as entrevistas com os gestores realizadas neste trabalho evidenciaram que o processo de certificação dos municípios pernambucanos para descentralizar a gestão das ações de vigilância epidemiológica evoluiu positivamente entre os anos de 2000 a 2011. Os achados apontaram para existência de três fases distintas na evolução temporal desse processo. Entre os anos de 2001 e 2003, pode-se indicar que foram certificados os municípios que já exerciam a gestão de grande parte das ações de vigilância epidemiológica ou que estavam mais preparados no que diz respeito aos parâmetros estabelecidos no protocolo de certificação.

O intervalo que se observou após 2003, com a estagnação da evolução da descentralização, coincidiu com o período de mudança na gestão municipal. Mesmo que não se possa afirmar ser esse o principal motivo dessa fase de menor atividade nas certificações, sabe-se que aquele momento de transição política pode ter exercido forte influência sobre a gestão do serviço público municipal de saúde, principalmente com relação ao desempenho e à estruturação de um sistema complexo como o de vigilância epidemiológica. Por fim, a partir de 2006, é possível perceber que as certificações voltaram a acontecer de forma mais expressiva, período este em que o processo de certificação foi modificado com o Pacto pela Saúde 12, passando a ocorrer por adesão da própria gestão municipal.

Observou-se que, em Pernambuco, não houve um padrão espacial definido de disseminação

Tabela 2

Proporção de óbitos com causa básica definida e variação percentual, segundo município de residência. Pernambuco, Brasil, $2001-2010$.

\begin{tabular}{|c|c|c|c|c|c|c|c|c|c|c|c|}
\hline \multirow[t]{2}{*}{ Municípios } & \multicolumn{10}{|c|}{ Ano } & \multirow{2}{*}{$\begin{array}{c}\text { Variação (\%) } \\
\text { 2001-2010 }\end{array}$} \\
\hline & 2001 & 2002 & 2003 & 2004 & 2005 & 2006 & 2007 & 2008 & 2009 & 2010 & \\
\hline Recife & 99,1 & 98,8 & 99,1 & 99,1 & 98,9 & 99,3 & 99,5 & 99,5 & 99,4 & 99,3 & 0,2 \\
\hline Limoeiro & 75,2 & 76,2 & 81,2 & 84,3 & 95,1 & 97,0 & 95,2 & 94,8 & 94,6 & 95,7 & 27,2 \\
\hline Palmares & 84,5 & 82,7 & 82,4 & 85,3 & 93,4 & 97,8 & 98,0 & 98,2 & 97,6 & 98,0 & 15,9 \\
\hline Caruaru & 78,1 & 78,6 & 80,0 & 85,3 & 89,2 & 90,1 & 92,6 & 92,6 & 91,2 & 90,9 & 16,3 \\
\hline Garanhuns & 56,4 & 62,6 & 64,7 & 71,0 & 69,6 & 77,1 & 95,5 & 88,5 & 87,2 & 90,0 & 59,7 \\
\hline Arcoverde & 76,2 & 78,2 & 70,3 & 72,3 & 81,0 & 91,4 & 92,6 & 88,2 & 91,9 & 95,4 & 25,2 \\
\hline Salgueiro & 90,8 & 88,8 & 87,1 & 85,6 & 87,1 & 91,1 & 91,6 & 96,1 & 96,8 & 94,6 & 4,2 \\
\hline Petrolina & 68,2 & 74,7 & 75,1 & 74,9 & 95,6 & 96,0 & 96,8 & 93,4 & 86,6 & 87,1 & 27,7 \\
\hline Ouricuri & 50,0 & 56,4 & 60,2 & 64,3 & 70,1 & 76,7 & 98,7 & 98,2 & 96,9 & 96,0 & 92,0 \\
\hline Afogados da Ingazeira & 54,1 & 57,6 & 65,2 & 74,2 & 83,4 & 95,4 & 94,8 & 97,8 & 97,1 & 95,1 & 75,8 \\
\hline Total no estado & 78,8 & 81,0 & 82,3 & 84,2 & 90,0 & 94,1 & 94,9 & 94,8 & 94,6 & 94,7 & 20,1 \\
\hline
\end{tabular}

Fonte: Sistema de Informações sobre Mortalidade (SIM)/Secretaria Executiva de Vigilância em Saúde da Secretaria de Saúde do Estado de Pernambuco. 
Tabela 3

Taxa de incidência de sífilis congênita (por 1.000 nascidos vivos) e variação percentual, segundo município de residência. Pernambuco, Brasil, $2001-2010$.

\begin{tabular}{|c|c|c|c|c|c|c|c|c|c|c|c|}
\hline \multirow[t]{2}{*}{ Municípios } & \multicolumn{10}{|c|}{ Ano } & \multirow{2}{*}{$\begin{array}{l}\text { Variação (\%) } \\
\text { 2001-2010 }\end{array}$} \\
\hline & 2001 & 2002 & 2003 & 2004 & 2005 & 2006 & 2007 & 2008 & 2009 & 2010 & \\
\hline Recife & 8,2 & 6,3 & 7,6 & 8,7 & 11,4 & 11,7 & 10,9 & 9,0 & 8,9 & 9,9 & 21,6 \\
\hline Limoeiro & 6,7 & 8,8 & 2,0 & 6,3 & 1,1 & 1,1 & 3,6 & 1,1 & 2,4 & 0,0 & $-100,0$ \\
\hline Palmares & 2,1 & 0,0 & 0,8 & 0,8 & 0,0 & 2,5 & 1,7 & 2,5 & 2,7 & 3,4 & 62,6 \\
\hline Caruaru & 5,6 & 3,7 & 6,0 & 5,3 & 5,3 & 7,0 & 4,0 & 2,7 & 1,7 & 1,9 & $-65,3$ \\
\hline Garanhuns & 0,0 & 0,0 & 0,4 & 0,0 & 0,4 & 3,4 & 0,0 & 0,4 & 0,8 & 0,4 & - \\
\hline Arcoverde & 2,7 & 2,1 & 3,6 & 0,8 & 3,3 & 2,3 & 2,6 & 0,0 & 0,8 & 0,0 & $-100,0$ \\
\hline Salgueiro & 0,8 & 0,0 & 0,0 & 1,8 & 0,9 & 1,9 & 1,0 & 2,0 & 3,6 & 1,9 & 122,1 \\
\hline Petrolina & 7,5 & 4,2 & 5,5 & 7,3 & 5,1 & 2,4 & 2,2 & 1,3 & 1,3 & 1,7 & $-77,5$ \\
\hline Ouricuri & 0,0 & 0,0 & 0,7 & 0,0 & 0,0 & 0,0 & 0,0 & 0,0 & 0,0 & 0,0 & 0,0 \\
\hline Afogados da Ingazeira & 0,0 & 0,0 & 0,0 & 0,0 & 0,0 & 1,7 & 0,0 & 0,0 & 0,0 & 0,0 & 0,0 \\
\hline Total no estado & 6,0 & 4,6 & 5,6 & 6,3 & 7,5 & 7,5 & 6,7 & 5,2 & 5,2 & 5,8 & $-3,3$ \\
\hline
\end{tabular}

Fonte: Sistema de Informação de Agravos de Notificação (SINAN) - Sistema de Informações sobre Nascidos Vivos (SINSAC)/Secretaria Executiva de Vigilância em Saúde da Secretaria de Saúde do Estado de Pernambuco.

do processo de descentralização da vigilância epidemiológica para gestão municipal. No ano 2000, três municípios-sede de Geres foram certificados (Petrolina, Caruaru e Recife), os quais estavam habilitados na Gestão Plena do Sistema Municipal de Saúde. Nos anos subsequentes, entre 2001 e 2003, os municípios de maior porte populacional e relevância econômica para o estado, ou seja, os demais municípios-sede e os municípios da Região Metropolitana do Recife, também logo foram certificados. Essa observação é coerente com os achados de um estudo que avaliou a vigilância epidemiológica e as características específicas da gestão municipal em Pernambuco no ano de 2003, e que concluiu que o porte populacional e a condição de gestão foram determinantes do grau de implantação da vigilância epidemiológica, uma vez que os municípios de grande porte e em Gestão Plena do Sistema de Saúde obtiveram melhor desempenho, ao passo que a localização geográfica não influenciou no resultado 13 .

Com efeito, é necessário discutir algumas questões relacionadas ao mecanismo de certificação da gestão das ações descentralizadas de vigilância epidemiológica adotado em Pernambuco. O instrumento utilizado para averiguação da capacidade instalada do município, composto de parâmetros tais como número de veículos, de salas, de computadores e de técnicos de nível médio/superior, realça a importância dada ao aspecto quantitativo em detrimento ao qualitativo no processo de descentralização da vigilância epidemiológica. Isso também pode ser percebido nas falas dos gestores entrevistados, que apontaram a deficiência na qualificação das equipes nas SMS, que não acompanharam a evolução da descentralização em grande parte dos municípios selecionados. Outro ponto a ser considerado é a forma verticalizada com que o processo de certificação dos municípios foi conduzido. Esse aspecto foi apontado pelos gestores como dificultador da descentralização, com destaque para a pactuação de ações e metas na PPI-ECD, as quais eram estabelecidas do nível federal de gestão para os estados, e destes para os municípios. Nos relatos dos gestores, foi citado que esse procedimento verticalizado contribuiu com a perda na qualidade da informação no início do processo, visto que não foram observadas as fragilidades e as particularidades do nível local.

Ressalta-se ainda que as vistorias realizadas com o propósito de verificar in loco os parâmetros estabelecidos pelo protocolo de certificação foram realizadas por diversas equipes, o que pode ter ocasionado a falta de padronização no processo. Na fala dos gestores, notou-se que, apesar da obrigatoriedade da estrutura mínima definida pelo instrumento de certificação, faltavam profissionais, estrutura física e insumos na vigilância epidemiológica, situação que persistiu após a descentralização. Esses relatos coincidem com os achados de Santos et al. ${ }^{14}$, os quais apontam para a precariedade da estrutura física e dos recursos humanos na vigilância epidemiológica de municípios baianos. Outros estudos destacam que falhas estruturais estão relacionadas diretamente com os impedimentos na descentraliza- 
ção das ações de vigilância epidemiológica para outros níveis do sistema, a saber: os níveis municipal, distrital e Unidade Básica de Saúde (UBS) 11,15,16. A fala do Gestor C no que diz respeito à real administração do FMS pode estar apontando para umas das causas dessa problemática citada.

Sabe-se que, apesar dos inegáveis avanços nas políticas de saúde no Brasil nos últimos anos, a gestão dos recursos humanos pouco avançou e é certamente a que convive com os maiores problemas e dificuldades devido, entre outros aspectos, à precariedade dos vínculos, contratações que não consideram as necessidades do setor, baixos salários e falta de um plano de cargos e carreira. Na vigilância epidemiológica esse quadro não é diferente, sendo apontado por diversos autores como um fator que obstaculiza a consolidação da vigilância nos sistemas municipais de saúde, tendo como consequência a insuficiência de equipes mínimas nos municípios para a execução das ações, as relações temporárias de trabalho e a falta de qualificação técnica 4,11,13.

As dificuldades na execução de algumas ações como, por exemplo, a investigação de óbitos, bem como a análise e disseminação da informação, mencionadas pelos gestores, também foram relatadas em outro estudo que avaliou o sistema de vigilância em saúde do Brasil e concluiu que os níveis estadual e municipal desse sistema estão mais aptos para detectar, avaliar e notificar do que para investigar, intervir e comunicar 17. Assim, ainda que o processo de descentralização propicie a aproximação do nível executor das ações à população e aos seus problemas de saúde, e que esta aproximação exija uma maior responsabilização por parte da gestão municipal, Carvalho et al. 13 indicam que o ato de assumir a execução das ações da vigilância epidemiológica tem demonstrado um caráter meramente descritivo, restrito à coleta de dados e, por vezes, à transmissão destes dados a outros níveis.

A ESF tem um papel articulador importante na ação local com os princípios basilares do SUS 18 e a sua integração com a vigilância epidemiológica no nível municipal é condição imprescindível para a construção da integralidade na atenção e para o alcance de resultados positivos 19,20. Dessa forma, apesar de a maioria dos gestores entrevistados neste trabalho referirem boa integração ESF-vigilância epidemiológica nos seus respectivos municípios, sabe-se que esta é uma das principais fragilidades que ainda persistem na consolidação do processo de descentralização das ações de vigilância epidemiológica 4,14,21,22 .

No que diz respeito à execução centralizada na SMS dos programas de tuberculose e hanseníase, é importante destacar um estudo realizado em municípios de uma microrregião mineira, que analisou o processo de descentralização das ações de controle da hanseníase para as unidades da ESF, e também encontrou essa realidade em alguns dos municípios da pesquisa e concluiu que os mesmos estavam em diferentes estágios de descentralização, de acordo com a especificidade local e engajamento dos gestores e dos profissionais de saúde. O estudo concluiu ainda que a integração das ações de prevenção e controle da hanseníase na Atenção Primária à Saúde é a melhor estratégia para alcançar o controle da doença em áreas endêmicas, uma vez que este processo está ancorado nos princípios da equidade e da acessibilidade 23 .

Por fim, objetivando analisar a evolução do processo de descentralização por meio do comportamento dos indicadores, observa-se que apenas a proporção de óbitos com causa básica definida apresentou crescimento expressivo nos municípios do estudo. Esse indicador tem fundamental importância como parâmetro de qualidade da informação sobre mortalidade, e a análise das séries históricas evidenciou melhoria no preenchimento da causa básica de morte nos municípios do estudo, bem como no estado como um todo. Outros trabalhos confirmam essa evidência 24,25 , sendo importante destacar ainda que esse indicador tem forte relação com o acesso à assistência médica no momento da morte. Assim, nos municípios de grande porte populacional, onde se concentra a maior parte dos serviços de saúde, a proporção de óbitos com causa básica definida é maior 25 .

Com relação ao indicador proporção de casos novos de tuberculose pulmonar bacilíferos encerrados pelo critério de cura, houve uma evolução positiva na maioria dos municípios estudados, evolução esta que não foi acompanhada pelo Estado de Pernambuco de um modo geral. Sabe-se que o controle da tuberculose está centrado principalmente na redução das fontes de infecção que só é obtida na medida em que os casos bacilíferos são detectados e curados, razão pela qual o acompanhamento desse indicador se torna importante. Dessa maneira, a variação positiva verificada pode indicar que pelo menos uma parte desse objetivo vem sendo alcançada naqueles municípios 26. É importante destacar, no entanto, que a análise dos indicadores proporção de casos novos de tuberculose pulmonar bacilíferos encerrados pelo critério de cura e taxa de incidência de sífilis congênita, deve ser feita com cautela, devido ao efeito gerado pelos pequenos números (número de casos) na variabilidade das taxas.

As oscilações observadas nos indicadores proporção de casos novos de tuberculose pulmonar bacilíferos encerrados pelo critério de cura 
e taxa de incidência de sífilis congênita, podem apontar para a existência de fragilidades no processo de descentralização da vigilância epidemiológica, sugerindo-se que a rotatividade dos recursos humanos e a desarticulação do processo de trabalho da vigilância epidemiológica com outros níveis do sistema, como por exemplo, hospital de referência, laboratório ou atenção básica tenham tido influência sobre esse resultado. Essa falta de linearidade na evolução de indicadores epidemiológicos também foi observada em um estudo que avaliou o impacto da descentralização das ações da vigilância epidemiológica para as unidades do Programa Saúde da Família (PSF) em um município baiano 26.

Vale ressaltar que certos entraves foram encontrados durante o desenvolvimento do presente trabalho. Alguns indicadores demonstrativos da responsabilização municipal a partir da descentralização da vigilância epidemiológica não foram utilizados devido à ausência de dados na série histórica completa.

Embora este estudo tenha apontado algumas deficiências na gestão e execução das ações de vigilância epidemiológica pela gestão municipal, a complexidade do objeto estudado requer um monitoramento contínuo deste processo. Assim, mesmo que a descentralização das ações de vigilância epidemiológica ainda seja incipiente em vários municípios, sabe-se que o papel de execu- tor das ações promove o empoderamento do nível municipal, quando o mesmo passa a produzir as informações necessárias à tomada de decisão. Diversos estudos evidenciam que a estratégia de descentralização das ações e dos recursos financeiros para o âmbito municipal tem proporcionado experiências bem-sucedidas, apontando para os efeitos positivos deste processo na vigilância epidemiológica 17,20.

Os resultados deste trabalho apontam para a necessidade do estabelecimento de um projeto político que assuma efetivamente a descentralização da vigilância epidemiológica como uma estratégia para a reorganização da atenção à saúde no nível municipal 4 . A adequação do perfil dos recursos humanos e do papel dos níveis estadual e regional torna-se condição indispensável para a descentralização do sistema. Esses profissionais precisam ser capacitados para a realização de supervisão e o acompanhamento dos municípios, propiciando-lhes autonomia na consolidação das informações, análise epidemiológica e elaboração de propostas voltadas à sua realidade 27. Finalmente, o processo de descentralização pode ser considerado politicamente irreversível, mas pode e deve ser tecnicamente viável, a depender de aprimoramentos administrativos em sua estrutura e de autêntica vontade política dos governantes 28 .

\section{Resumen}

El objetivo del estudio fue analizar la relación entre el proceso de descentralización del Sistema Único de Salud (SUS) y el desarrollo de acciones de vigilancia epidemiológica en los municipios de Pernambuco, Brasil. La estrategia de trabajo fue un estudio exploratorio descriptivo, cualitativo y cuantitativo, con la realización de una revisión documental, entrevistas semi-estructuradas con informantes clave, y un estudio ecológico espacial con una serie de tiempo de 10 años (2001-2010) de indicadores seleccionados. El estudio mostró que el proceso de descentralización en Pernambuco tiene adhesión y evolucionó, pero con la existencia de desigual- dades y debilidades en su desarrollo, revelado también por la oscilación de la serie histórica de los indicadores. A pesar de que el proceso de descentralización de la vigilancia epidemiológica es aún incipiente en algunos municipios, se sabe que el papel de ejecutor de acciones promueve el empoderamiento del nivel municipal, cuando se comienza a producir información necesaria para la toma de decisiones.

Vigilancia Epidemiológica; Descentralización; Administración Municipal 


\section{Colaboradores}

A. C. Albuquerque concebeu e planejou a pesquisa, levantou e analisou os dados e redigiu o artigo. E. L. A. Mota e E. Felisberto participaram da concepção e planejamento da pesquisa e colaboraram na redação, análise e revisão crítica do artigo.

\section{Referências}

1. Brasil. Lei no 8.080 , de 19 de setembro de 1990 . Dispõe sobre as condições para a promoção, proteção e recuperação da saúde, a organização e funcionamento dos serviços correspondentes e dá outras providências. Diário Oficial da União 1990; 20 set.

2. Ministério da Saúde. Portaria no 1.399 , de 15 de dezembro de 1999. Regulamenta a NOB SUS 01/96 no que se refere às competências da União, estados, municípios e Distrito Federal, na área de epidemiologia e controle de doenças, define a sistemática de financiamento e dá outras providências. Diário Oficial da União 1999; 16 dez.

3. Ministério da Saúde. Portaria no 950, de 23 de dezembro de 1999. Define os valores anuais per capita e por quilômetro quadrado, relativos aos recursos federais destinados à composição do Teto Financeiro de Epidemiologia e Controle de Doenças de cada um dos estratos previstos no art. 14 da Portaria MS no 1.399/99. Diário Oficial da União 1999; 24 dez.

\section{Agradecimentos}

Agradecemos à Secretaria Executiva de Vigilância em Saúde da SES-PE, que colaborou com a realização desta pesquisa.
4. Santos SSBS, Melo CMM. Avaliação da descentralização da vigilância epidemiológica para a Equipe de Saúde da Família. Ciênc Saúde Coletiva 2008; 13:1923-32.

5. Secretaria de Vigilância em Saúde, Ministério da Saúde. Guia de vigilância epidemiológica. 7a Ed. Brasília: Ministério da Saúde; 2009.

6. Fracolli LA, Faria LS, Pereira JG, Melchior R, Bertolozzi MR. Vigilância à saúde: deve se constituir como política pública? Saúde Soc 2008; 17:184-92.

7. Silva Junior JB. Epidemiologia em serviço: uma avaliação de desempenho do Sistema Nacional de Vigilância em Saúde [Tese de Doutorado]. Campinas: Faculdade de Ciências Médicas, Universidade Estadual de Campinas; 2004.

8. Costa JMBS, Felisberto E, Bezerra LCA, Cesse EAP, Samico IC. Monitoramento do desempenho da gestão da vigilância em saúde: instrumento e estratégias de uso. Ciênc Saúde Coletiva 2013; 18:1201-16 
9. Bardin L. Análise de conteúdo. Lisboa: Edições 70; 1979.

10. Minayo MCS. O desafio do conhecimento: pesquisa qualitativa em saúde. São Paulo: Hucitec; 2010.

11. Bezerra LCA, Freese E, Frias PG, Samico I, Almeida CKAA. Vigilância epidemiológica no âmbito municipal: avaliação do grau de implantação das ações. Cad Saúde Pública 2009; 25:827-39.

12. Ministério da Saúde. Portaria no 399, de 22 de fevereiro de 2006. Divulga o Pacto pela Saúde 2006 - Consolidação do SUS e aprova as Diretrizes Operacionais do referido Pacto. Diário Oficial da União 2006; 23 fev.

13. Carvalho EF, Cesse EAP, Albuquerque MIN, Albuquerque LC, Dubeux LS. Avaliação da vigilância epidemiológica em âmbito municipal. Rev Bras Saúde Matern Infant 2005; 5 Suppl 1:53-62.

14. Santos SSBS, Melo CMM, Costa HOG, Tanaka OU, Ramos FM, Santana MCC, et al. Avaliação da capacidade de gestão descentralizada da vigilância epidemiológica no Estado da Bahia. Ciênc Saúde Coletiva 2012; 17:873-82.

15. Villa TCS, Palha PF, Muniz JN, Cardozo-Gonzales RI, Pinto Neto JM, Assis MMA. A vigilância epidemiológica e a perspectiva de trabalho no território: Secretaria Municipal de Saúde - Ribeirão Preto. Rev Latinoam Enferm 2002; 10:21-7.

16. Passos IMR. "Assistir" e "vigiar" as ações de vigilância epidemiológica nas unidades básicas de saúde. Avanços e perspectivas [Tese de Doutorado]. Ribeirão Preto: Escola de Enfermagem de Ribeirão Preto, Universidade de São Paulo; 2003.

17. Teixeira MG, Costa MCN, Souza LPF, Nascimento EMR, Barreto ML, Barbosa N, et al. Evaluation of Brazil's public health surveillance system within the context of the International Health Regulations (2005). Rev Panam Salud Pública 2012; 32: 49-55.

18. Bossert T, Larranaga O, Meir FR. Decentralization of health systems in Latin America. Rev Panam Salud Pública 2000; 8:84-92.

19. Ministério da Saúde. Portaria no 3.252, de 22 de dezembro de 2009. Aprova as diretrizes para execução e financiamento das ações de Vigilância em Saúde pela União, Estados, Distrito Federal e Municípios e dá outras providências. Diário Oficial da União 2009; 23 dez.
20. Barbosa MCL, Costa MC, Teixeira MG, Mota ELA, Pereira SM. Efeitos da descentralização das ações de vigilância epidemiológica para as equipes de saúde da família. Epidemiol Serv Saúde 2010; 19:347-54.

21. Leite JA, Assis MMA, Cerqueira EM. Vigilância epidemiológica no sistema local de saúde. Rev Bras Enferm 2003; 56:178-83.

22. Cerqueira EM, Assis MMA, Villa TCS, Leite JA. Vigilância epidemiológica no processo de municipalização do Sistema de Saúde em Feira de SantanaBA. Epidemiol Serv Saúde 2003; 12:213-23.

23. Lanza FM, Lana FCF. Descentralização das ações de controle da hanseníase na microrregião de Almenara, Minas Gerais. Rev Latinoam Enferm 2011; 19:187-94.

24. Frias PG, Pereira PMH, Andrade CLT, Lira PIC, Szwarcwald CL. Avaliação da adequação das informações de mortalidade e nascidos vivos no Estado de Pernambuco, Brasil. Cad Saúde Pública 2010; 26:671-81.

25. Rodrigues M, Bonfim C, Frias PG, Braga C, Gurgel IGD, Medeiros Z. Diferenciais na adequação das informações de eventos vitais nos municípios de Pernambuco, 2006-2008. Rev Bras Epidemiol 2012; 15:275-84.

26. Barbosa MCL. Avaliação do impacto da descentralização de ações de vigilância epidemiológica para as unidades de saúde da família sobre o desempenho de alguns indicadores [Dissertação de Mestrado]. Salvador: Instituto de Saúde Coletiva, Universidade Federal da Bahia; 2008.

27. Schout D. Municipalização da vigilância epidemiológica: uma proposta em construção. Saúde Soc 1994; 3:11-28.

28. Villela MM, Souza JMB, Melo VP, Dias JCP. Vigilância epidemiológica da doença de Chagas em programa descentralizado: avaliação de conhecimentos e práticas de agentes municipais em região endêmica de Minas Gerais, Brasil. Cad Saúde Pública 2007; 23:2428-38.

Recebido em 03/Jul/2014

Versão final reapresentada em 07/Out/2014

Aprovado em 01/Dez/2014 